Vivre, penser, écrire en exil

\title{
Les « expulsions volontaires » : un mode de gouvernement des « étrangers indésirables »?
}

"Voluntary Deportations": A Technique of Government of Unwanted Foreigners? ¿Las «expulsiones voluntarias»: un modo de gubernamentalidad de los extranjeros indeseables?

Clément de Senarclens et Ibrahim Soysüren

\section{(2) OpenEdition}

Journals

\section{Édition électronique}

URL : https://journals.openedition.org/remi/8539

DOI : $10.4000 /$ remi.8539

ISSN : $1777-5418$

Éditeur

Université de Poitiers

\section{Édition imprimée}

Date de publication : 1 mars 2017

Pagination : 133-156

ISBN : 979-10-90426-30-6

ISSN : 0765-0752

Référence électronique

Clément de Senarclens et Ibrahim Soysüren, « Les « expulsions volontaires » : un mode de gouvernement des «étrangers indésirables »? », Revue européenne des migrations internationales [En ligne], vol. $33-\mathrm{n}^{\circ} 1$ | 2017, mis en ligne le 01 mars 2019, consulté le 15 avril 2022. URL : http:// journals.openedition.org/remi/8539; DOI : https://doi.org/10.4000/remi.8539 


\title{
Les " expulsions volontaires " : un mode de gouvernement des "étrangers indésirables "?
}

\author{
Clément de Senarclens ${ }^{1}$ et \\ Ibrahim Soysüren ${ }^{2}$
}

\section{Introduction}

L'expulsion ${ }^{3}$ des requérants d'asile déboutés est devenue un sujet de préoccupation croissante au sein des pays européens à partir du début des années 1980 (Gibney et Hansen, 2003) ${ }^{4}$. L'augmentation du nombre de requérants déboutés et les difficultés liées à l'exécution de leur renvoi ont amené les États européens à élaborer divers instruments et stratégies visant à faciliter la mise en œuvre de l'éloignement de cette nouvelle catégorie d'étrangers "indésirables " (Noll, 1999). Parmi ceux-ci, figure le recours à la contrainte physique au travers de l'usage de la détention administrative et des procédures de renvoi. Les difficultés d'ordre pratique (liées à l'identification et à l'obtention de documents de

\footnotetext{
1 Doctorant, Centre de droit des migrations, Université de Neuchâtel, Rue Abram-Louis Breguet 1, 2000 Neuchâtel, Suisse ; clement.desenarclens@unine.ch

2 Doctorant, Institut de Sociologie, Université de Neuchâtel, Faubourg de I'Hôpital 27, 2000 Neuchâtel, Suisse; ibrahim.soysuren@unine.ch

3 Selon le cadre juridique suisse, l'usage du terme d'expulsion devrait être limité aux décisions d'éloignement prononcées pour des motifs politiques liés à la sécurité intérieure ou extérieure de la Suisse au titre de l'article 68 de la loi fédérale sur les étrangers du 16.12.2005 (RS 142.20). Les autres formes d'éloignement devraient, selon cette acception, être qualifiées de "renvoi ". Du fait de l'euphémisme que comporte ce terme, nous avons généralement préféré employer le terme d' "expulsion " pris dans son sens général. Nous avons toutefois conservé les termes juridiques, tels que " décision de renvoi ", " exécution de renvoi " ou " procédure de renvoi " qui ont des sens spécifiques dans le droit suisse.

4 Cet article est le fruit d'une collaboration entre deux projets de recherche financés par le Fond National Suisse de recherche scientifique (FNS) : "L'expulsion des sanspapiers et des étrangers délinquants. Pour une sociologie comparative de l'expulsion des étrangers indésirables en Suisse, France et Turquie ", dirigé par François Hainard et "Migration and Security in Switzerland ", dirigé par Christin Achermann.
} 
voyage), le coût très élevé de ces mesures $^{5}$, ainsi que les nombreuses critiques qu'elles soulèvent au sein de la société civile ont toutefois amené les États à développer un certain nombre de politiques et de dispositions afin de favoriser les départs dits "volontaires ". Cette préoccupation des pays de l'Union européenne (UE) ressort clairement des textes juridiques européens, tels que la Directive du 16 décembre 2008 relative aux normes et procédures communes applicables dans les États membres au retour des ressortissants de pays tiers en séjour irrégulier (2008/115/CE). Ce texte, transposé dans le droit des pays membres et pays associés comme la Suisse ${ }^{6}$, oblige à accorder un délai de départ " volontaire " d'au moins sept jours (sauf exception) aux étrangers qui font l'objet d'une décision d'expulsion. En tant que pays associé à I'UE par le biais des accords bilatéraux, le cas de la Suisse, sur lequel porte notre analyse, ne constitue donc pas, loin de là, une exception. Bien que cela n'entre pas en droite ligne de l'objectif du présent article, cette contribution pourra, dans un deuxième temps, servir de base afin de mettre en lumière d'éventuelles tendances d'uniformisation des discours et des pratiques d'expulsion à l'échelle européenne ${ }^{7}$.

Diverses contributions ont remis en question le caractère "volontaire " des départs ayant lieu dans le cadre de différents " programmes d'assistance au retour ", en soulignant l'absence de décision librement consentie de la part des individus rentrant dans ce cadre (Gibney et Hansen, 2003 ; Blitz et al., 2005 ; Webber, 2011). Cet article propose d'effectuer un pas de plus dans cette direction en mettant en relief la manière dont les mesures d'incitations offertes dans le cadre de ces programmes s'articulent avec diverses formes de contraintes (juridiques, matérielles, psychologiques et physiques). Cette articulation aurait selon notre analyse pour but d'amener les requérants déboutés à se plier à l'obligation légale de quitter le territoire qui pèse sur eux, sans qu'il soit fait un recours effectif à la contrainte physique.

Nous proposons de nommer ces départs, et les politiques qui les soustendent, par le terme d' "expulsion volontaire " afin de faire ressortir le caractère paradoxal d'un discours qui qualifie de "volontaire " le départ d'étrangers soumis à l'obligation légale de quitter le territoire et, où la potentialité du recours à la contrainte physique joue un rôle prépondérant. Cette notion permet également d'illustrer l'articulation existant entre les incitations matérielles des " programmes d'assistance au retour volontaire " et les diverses formes de

\footnotetext{
5 Les chiffres démontrent, en effet, que le recours à la contrainte physique lors de la procédure de renvoi est une pratique particulièrement coûteuse. Selon un article du Temps, la Suisse dépense 200000 CHF (192 $000 €)$ pour un vol spécial vers l'Afrique australe et l'ancien Office fédéral des migrations (ODM), désormais dénommé Secrétariat d'État aux migrations (SEM) depuis le 1er janvier 2015, parle d'un coût moyen variant entre $7000 \mathrm{CHF}(6700 €)$ et $10000 \mathrm{CHF}(9600 €)$ par personne expulsée (Des expulsions forcées délicates et coûteuses, Le Temps, 02/10/2010).

6 Voir Arrêté fédéral portant sur l'approbation et la mise en œuvre de l'échange de notes entre la Suisse et la CE concernant la reprise de la directive CE sur le retour (directive 2008/115/CE) (Développement de I'acquis de Schengen) du 18 juin 2010 (Recueil Officiel $2010: 5925)$.

7 Cela constitue d'ailleurs l'objet d'une recherche en cours, financée par le FNS, menée par Ibrahim Soysüren sous la direction de Mihaela Nedelcu, intitulée « Usages institutionnels des instruments technologiques européens d'identification et stratégies de contournement des étrangers à expulser en France et en Suisse. Vers une européanisation de I'expulsion des étrangers indésirables ? ".
} 
contraintes qui président à ces départs. Nous désignerons d' « étrangers indésirables " les ressortissants étrangers soumis à une obligation légale de quitter le territoire ${ }^{8}$. Si la présente contribution se focalise sur la catégorie des requérants d'asile déboutés, l'un de nos objectifs vise cependant d'offrir un cadre d'analyse qui pourra également servir à l'étude de cas d'autres catégories de migrants.

Les politiques et mesures visant à l'exécution du renvoi des requérants déboutés seront analysées à l'aide du concept de " gouvernementalité ", élaboré par Foucault (1994a ; 1994b ; 2004), puis repris et développé par divers auteurs au sujet des politiques migratoires (voir notamment Walters, 2002 ; Fassin, 2011 ; Geiger et Pécoud, 2013 ; Inda et Dowling, 2013). La gouvernementalité, ou " art de gouverner " a été définie comme l'étude des formes plus ou moins réfléchies et systématiques de penser et d'agir qui ont pour but d'influer, de réguler ou de contrôler les comportements d'individus (Inda et Dowling, 2013). Bien que son développement soit considéré dans un cadre plus général, le concept de gouvernementalité apparaît adapté afin de mettre en évidence la manière d'articuler les diverses formes de contrainte et d'incitation dans l'optique de pousser les individus à se conformer "d'eux-mêmes " aux injonctions des autorités. Ce concept amène en effet une " correction " ou un " raffinement " (Bröckling et al., 2011) dans une analyse foucaldienne du pouvoir " qui dépasse la problématique du consensus et de la volonté, d'une part, et de la conquête et de la guerre, d'autre part " et permet ainsi " d'étudier la capacité de l'individu "autonome" à se contrôler " (Lemke, $2004: 20)$. Le concept de gouvernementalité nous permettra $\mathrm{d}^{\prime}$ " explorer les rapports entre les techniques de soi et les techniques de domination " (Lemke, $2004: 20)$.

II convient de signaler dès à présent que l'objet de cet article n'est pas tant d'évaluer l'efficacité des mesures mises en œuvre afin d'exécuter le renvoi $d^{\prime}$ " étrangers indésirables ", que de commencer par mettre en évidence la manière dont cette politique est pensée par l'administration. Selon Foucault (1994a), la première étape nécessaire à la remise en cause d'une technique de gouvernement consiste en effet en un travail d'objectivation. Si selon lui, l'absence de formulation des rationalités de gouvernement rend difficile leur remise en question, c'est dans notre cas l'existence d'un discours officiel très élaboré, qui rend la remise en question de ces politiques particulièrement ardue.

Afin de réaliser ce travail d'objectivation, nous commencerons donc par décrypter les discours et catégories de la politique suisse d'exécution des renvois, tels qu'ils ressortent de l'étude de divers matériaux (discours et rapports officiels sur la "politique de retour ", site Internet de l'administration suisse, articles de journaux et émissions de télévision, etc.). Cette première partie mettra en évidence le dispositif par lequel ce discours crée une catégorisation binaire entre " retours volontaires " et " contraints ", qui légitime le recours à la contrainte physique, tout en minimisant l'usage qui en est fait. Dans un deuxième temps, nous confronterons ces discours au processus de mise en œuvre de ces politiques dans la pratique à la lumière de l'analyse des textes juridiques et administratifs, d'entretiens réalisés avec des requérants d'asile

8 Nous avons toutefois décidé de reprendre cette catégorie (en l'entourant toutefois des guillemets pour indiquer clairement qu'il ne s'agit pas là d'une catégorie analytique) pour illustrer la manière dont ces individus sont considérés par les autorités. 
déboutés et des membres des administrations responsables de l'exécution des renvois. Cela nous permettra de remettre en question le discours préalablement analysé, en démêlant la manière dont s'articulent dans la pratique les différentes formes d'incitation et de contraintes (légales, matérielles, psychologiques et physiques) placées sur les requérants déboutés. Cette analyse nous permettra de faire ressortir en guise de conclusion un mode de gouvernement des " étrangers indésirables " que nous qualifierons d'« expulsions volontaires ".

\section{Discours et catégories de la " politique de retour " suisse}

La politique d'exécution des renvois plus connue en Suisse sous le terme de "politique de retour " comprend diverses mesures de contrainte et d'incitation visant en particulier le départ des requérants d'asile déboutés. Suivant le discours émanant du gouvernement, cette politique se fonderait en premier lieu sur des mesures d'incitation visant à favoriser des retours qualifiés de " volontaires " ou " autonomes " lorsque le départ a lieu sans recours direct à des formes de contrainte physique. Toutefois, lorsque - malgré l'injonction de quitter le territoire et l'offre $d^{\prime}$ " aide au retour " - la personne refuse de quitter le territoire, les autorités seraient alors obligées de recourir à la contrainte.

Ainsi, lors d'un symposium consacré au droit et à la politique suisse des migrations, la directrice adjointe de l'Office fédéral des migrations (ODM) ${ }^{9}$, présentait la "politique suisse de retour " comme étant fondée sur deux principales composantes ${ }^{10}$. La première consisterait en la promotion des retours " volontaires " au travers de mesures d'incitation. Ces mesures incitatives détaillées dans la partie suivante - comprennent une somme d'argent et un projet de réinsertion qui peut être financé dans le pays d'origine par l'intermédiaire de I'Organisation Internationale pour les Migrations (OIM).

La seconde composante consisterait dans le recours à la contrainte au travers de l'usage de la détention administrative et des procédures de renvoi sous contraintes. Selon le discours entourant cette politique, il ne serait fait usage de forme de contraintes qu'en dernier recours dans le cas où les requérants déboutés refuseraient obstinément de collaborer avec les autorités. Dans ce contexte, la " contrainte " n'est toutefois entendue que sous ses formes les plus manifestes, à savoir la contrainte physique ayant cours dans le cas de la détention administrative ou de l'exécution des procédures " retour sous contrainte".

Il est ainsi souvent réaffirmé par les autorités qu'il ne serait fait recours à la " contrainte " qu'en ultime recours, lorsque la personne concernée refuserait de quitter " volontairement " le territoire. Ainsi, lors d'un entretien paru dans $L e$ Temps, la ministre Simonetta Sommaruga en charge des questions de migration

9 Cet organisme fédéral a été baptisé depuis le début 2015 Secrétariat d'État aux migrations. Comme le matériel sur lequel s'appuie cet article est plus ancien, afin de ne pas créer de confusion pour le lecteur nous avons continué à utiliser son ancien nom.

10 Gugger Bruckdorfer Evelyne (2011) Vollzug der Wegweisung: Aktueller Stand und Perspektiven aus Sicht des BFM, 7e journées du droit suisse des migrations, 5-6 septembre 2011. 
affirmait que : "C'est la pire des situations pour tout le monde et surtout pour les principaux concernés. [...] II ne faut pas oublier que ces vols spéciaux n'interviennent qu'en dernier recours : un requérant débouté a d'abord le choix de rentrer de son plein gré, avec un soutien financier de la Confédération " ${ }^{11}$.

Par ailleurs, il est également fréquemment rappelé que le nombre de " départs forcés" - en comparaison avec les départs pour lesquels les autorités n'ont pas à faire recours à la contrainte physique - demeure en réalité très faible.

Graphique 1 : Éloignements par voie aérienne 2010-2013

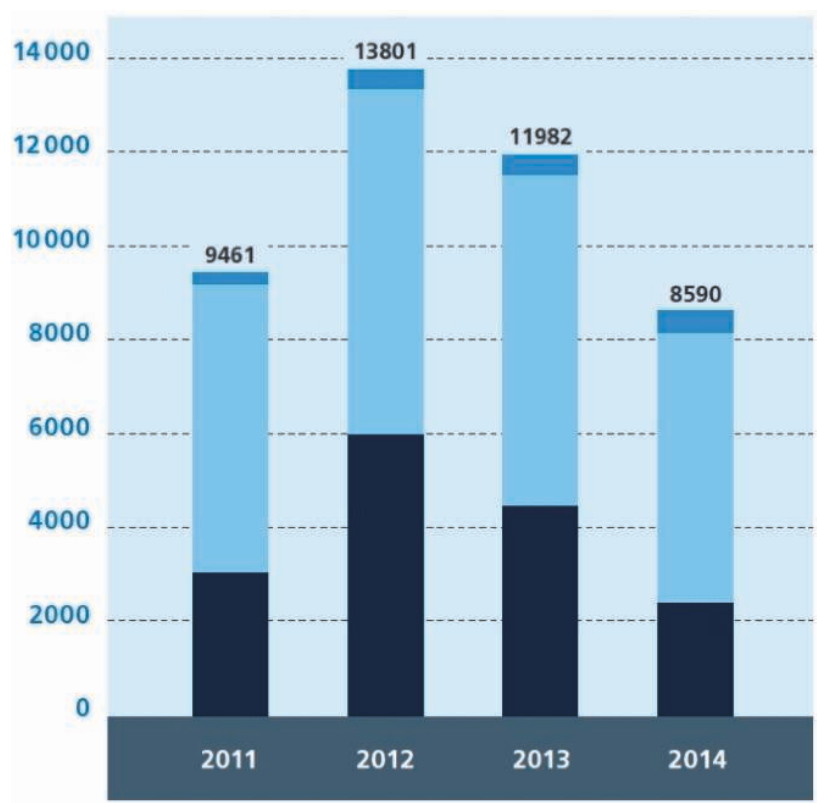

Rapatriement sous escorte policière jusqu'au pays de destination Rapatriements de niveau 1

Départs volontaires

Source : Rapport sur la migration 2014 (ODM, 2014 : 29).

11 Renvois forcés : “ La solution idéale n'existe pas », entretien avec Simonetta Sommaruga, ministre de Justice et Police, Le Temps, 17/09/2011. 
Au-delà de la réalité factuelle de cette constatation, illustrée dans le graphique repris du rapport annuel de I'ODM (2014: 29), I'insistance sur le faible pourcentage de personnes expulsées dans le cadre de "vols spéciaux " est employée afin de suggérer que la contrainte physique serait un "mal nécessaire ", qui occuperait fort heureusement une place marginale au sein de la politique de retour.

Bien que le recours direct à des formes de contraintes physiques apparaisse en effet relativement limité, nous arguerons ici que c'est en réalité la potentialité d'y faire recours qui représente, au fond, la pierre angulaire de la politique de retour. Cela ressort en fait très explicitement de diverses déclarations des membres de l'administration affirmant que la politique de retour s'effondrerait sans la possibilité du recours à la force physique pour les personnes refusant de partir d'elles-mêmes. La ministre Sommaruga déclarait ainsi, lors d'une émission télévisée : "Si vous n'avez pas ce tout dernier élément [les vols spéciaux], le système s'écroule $"{ }^{12}$. Le fait que la politique de retour ne puisse se maintenir en l'absence de formes les plus extrêmes de contraintes démontre ainsi bel et bien l'importance que revêt la contrainte pour l'ensemble des retours ayant lieu dans ce cadre. Ainsi, même si le recours effectif aux formes les plus extrêmes de coercition physique apparaît relativement restreint, cela ne devrait pas pour autant amener à négliger que la coercition physique - par les autres formes de contraintes qui en découlent - constitue effectivement le fondement de cette politique.

En résumé, le discours officiel institue une dichotomie des formes de " retours " pouvant avoir lieu dans le cadre de cette politique. II existerait, d'une part, des " retours volontaires " lorsque les personnes rentreraient dans le cadre de l'aide au retour ou sans que les autorités aient à intervenir et, de l'autre, des "retours contraints " lorsque les autorités auraient à faire recours à la contrainte physique. Ces catégories permettent, selon nous, de véhiculer un discours " positif " à l'égard de la politique de retour en minimisant le rôle joué par la coercition physique, et en masquant les autres formes de contraintes qui sont à l'œuvre lors de l'exécution de décisions de renvoi présentée comme en tant que " retour volontaire ".

Avant de passer en revue les diverses formes de contraintes à l'œuvre lors des différentes procédures d'expulsion et de souligner comment celles-ci s'articulent pour former une technique de gouvernement des " étrangers indésirables ", nous présenterons d'abord brièvement la catégorisation de ces différentes formes d'expulsion, non plus au sein du discours officiel auprès du public, mais au sein même de l'administration et des textes légaux. La prise en compte des différences dans les critères employés afin de fonder les diverses catégories nous permettra ainsi de mieux comprendre les enjeux qu'elles soustendent.

12 Simonetta Sommaruga lors de l'émission Infrarouge après la diffusion du film « Vol spécial ", TSR1, 28/03/2012. 


\section{Catégorisation légale des différentes formes de retour}

Si comme nous venons de le voir, une catégorisation binaire entre " retours volontaires " et " contraints " est opérée dans le discours du gouvernement, les textes légaux opèrent quant à eux des distinctions supplémentaires. Avant même d'entrer dans une analyse plus approfondie des qualificatifs employés, on peut déjà noter qu'il est fait référence au terme de " départ " que de " retour " pour caractériser les procédures d'exécution d'une décision de renvoi. Bien que ce premier élément puisse a priori paraître anodin, il indique toutefois que le focus premier de la politique porte clairement sur le départ du territoire suisse, et non sur le processus de retour dans le pays d'origine.

En ce qui concerne maintenant la manière de qualifier ces départs, on constate d'abord une différence, inexistante dans le discours à destination du public, établie entre des départs "volontaires" et " autonomes ". Ainsi, I'ancien chef de la "Section Aide au retour " de I'ODM précisait que : "Un départ volontaire, dans l'acceptation suisse, correspond à un retour dans le pays d'origine exempt d'obligation juridique, les étrangers en question ayant un statut qui leur donne le droit de séjourner en Suisse. Le départ est considéré comme autonome lorsque le droit de séjourner en Suisse expire et que l'étranger en question quitte le pays dans le délai prescrit et sans que l'on doive recourir à des mesures policières " (Kaser et Schenker, 2008 : 211). On constate donc que dès le moment où des personnes sont soumises à une obligation légale de quitter le territoire, il ne devrait plus être possible de qualifier leur retour (ou plutôt leur départ) de volontaire. Mais cela pourrait au mieux être considéré comme " autonome " dans le cas où il n'est pas fait recours à des mesures de contraintes (entendue selon l'acception légale restreinte de coercition physique).

L'Ordonnance relative au financement de l'asile précise par ailleurs, à I'article 62, que "Par retour autonome, on entend tout départ de Suisse effectué par une personne de son propre chef ou suite à une décision de renvoi prononcée à son égard ". La catégorie de "retour volontaire " dont il est aisément fait recours dans le débat public se rapporte par conséquent plutôt en terme légal à celle de " départ autonome ", qui met en avant l'absence de contrainte physique lors de l'exécution de la décision de renvoi.

On peut d'ores et déjà remarquer que si le discours public, par son recours à la catégorie de "retour volontaire ", focalise l'attention sur la part de choix (ou de décision librement consentie) que comporte un départ où il n'est pas fait un recours direct à la coercition physique, les catégorisations légales et administratives se concentrent, quant à elles, plus pratiquement sur le degré de contrainte nécessaire à l'exécution du renvoi. L'Ordonnance relative à l'usage de la contrainte (OLUsC) liste ainsi, à l'article 28, les quatre niveaux de contrainte qui peuvent être déployés lors de l'exécution des renvois. 
Encadré 1 : « Niveaux d'exécution des rapatriements " selon article 28 de l'Ordonnance sur l'usage de la contrainte et de mesures policières dans les domaines relevant de la compétence de la Confédération (OLUsC, R.S. 364.3) ${ }^{13}$

\begin{tabular}{|c|}
\hline $\begin{array}{l}1 \text { L'autorité ordonne les modalités du rapatriement en } \\
\text { fonction du comportement probable de la personne à } \\
\text { transporter et des circonstances concrètes. Les niveaux } \\
\text { d'exécution des rapatriements suivants sont prévus : } \\
\text { Niveau } 1 \text { : la personne à rapatrier a donné son accord } \\
\text { à un retour autonome ; elle est escortée par la police jusqu'à } \\
\text { l'embarquement, mais poursuit son voyage seule ; } \\
\text { Niveau } 2 \text { : la personne à rapatrier n'a pas donné son accord } \\
\text { à un retour autonome ; elle est en principe escortée par } \\
\text { deux agents de police en civil. Au besoin, elle est menottée ; }\end{array}$ \\
\hline
\end{tabular}

II ressort donc de l'analyse des catégories légales portant sur l'exécution des décisions de renvoi que le cadre juridique catégorise les départs, non pas en fonction du caractère librement consenti ou contrainte de la décision de I'individu à se plier à l'injonction qui lui est faite de quitter le territoire, mais du degré de coercition physique auquel les autorités font recours afin d'exécuter l'expulsion.

La citation suivante permet de remarquer comment le discours des fonctionnaires en charge de l'exécution des renvois cherche à faire le lien entre le degré de consentement ou de résistance qu'oppose I'individu à son obligation de quitter le territoire, et le degré croissant de contrainte auquel l'État fera recours.

" Ça peut arriver que la personne dise: "OK, j'ai pris note de mon obligation de quitter la Suisse", et puis [qu'elle] parte de manière volontaire, sans intervention aucune de nos services. Ce n'est pas très souvent, mais ça peut arriver. Le stade suivant, c'est de convoquer la personne ici et de faire un entretien de départ. On va lui expliquer qu'il doit quitter la Suisse, que la décision est en force et exécutoire. Voir si on arrive à le

faire adhérer, à le faire collaborer à ce renvoi-là, à ce départ-là, voire même lui proposer une aide au retour, la possibilité de financer un projet, de donner un peu d'argent pour faciliter la réintégration dans le pays. [...] Et puis, si cette personne ne collabore pas,

13 À noter que le terme de rapatriement ne correspond pas à la définition donnée par I'OIM (2007: 68) : "Opération consistant à assurer le, retour de réfugiés, de prisonniers de guerre et d'internés civils sur le territoire de leur État d'origine ". L'usage qui en est fait ici semble être guidé par le souci d'utiliser les termes les moins choquants possible. Ainsi au lieu d'expulsion, renvoi ou éloignement forcé, l'ordonnance parle de rapatriement.

14 Dû aux réticences des compagnies aériennes et des résistances de la part des passagers de voyager en présence de personnes entravées, ce niveau n'est de fait pas appliqué. 
alors, on peut envisager de faire un départ accompagné, forcé, de raccompagner ces gens dans leur pays sur des vols de ligne normaux avec un accompagnement policier ou au moins les amener à la frontière, enfin à l'aéroport pour qu'il prenne l'avion. Et puis, si ça ne marche toujours pas, s'il refuse de prendre l'avion, de partir, on peut envisager les mesures de contrainte. Donc, on a une graduation dans le processus pour faire quitter la

Suisse " (Responsable d'un service cantonal de migration, entretien du 12 juin 2013).

Le discours des fonctionnaires en charge des renvois continue à alimenter la confusion existant au sein du discours public entre les départs "volontaires" ou les individus décident librement de quitter le territoire sans être soumis à une décision de renvoi - et les départs qui devraient être qualifiés d' " autonomes " dans le cas où le départ intervient sans que les autorités aient à faire usage de la force. On remarque également l'idée du recours graduel à la contrainte que feraient les autorités en fonction du comportement de la personne concernée.

"C'est toujours l'individu qui s'oppose au renvoi et qui nous pousse à passer au degré supérieur et puis on va arriver jusqu'au vol spécial à cause de ça "

(Responsable d'un service cantonal de migration, entretien du 28 mai 2013).

Comme cela ressort particulièrement clairement de cette dernière citation, ce discours permet également de légitimer l'usage de la force auquel les autorités " devraient " faire recours en rejetant la responsabilité sur le comportement de I'individu.

\section{L'articulation des formes de contraintes et d'incitation en vue du gouvernement des étrangers indésirables}

Nous avons jusqu'ici mis en évidence la manière dont la politique suisse de retour est légitimée au travers de discours et des catégories discursives, administratives et légales sur lesquels elle repose. En nous fondant sur des observations de terrain et des entretiens effectués avec des requérants déboutés et des membres de l'administration, nous allons à présent mettre en évidence le décalage existant entre ces discours et le processus de mise en œuvre de cette politique. Cette analyse nous permettra de mieux saisir les formes de contraintes juridiques, matérielles, psychologiques et physiques s'exerçant sur les requérants déboutés et les modalités de leur articulation avec les incitations au départ proposées dans le cadre de l'aide au retour.

Nous commencerons par présenter les diverses formes de contraintes exercées à l'encontre des requérants déboutés de manière distincte. Ce découpage de la réalité observée est toutefois d'ordre principalement heuristique, puisque nous arguerons en conclusion que c'est précisément sur la base des liens entre ces diverses formes de contraintes et d'incitations que les autorités se fondent pour tenter de gouverner les conduites de cette catégorie d'étrangers.

\section{Incitations matérielles au retour}

Au-delà de l'organisation et de la prise en charge des frais du voyage, les prestations offertes dans le cadre des programmes dits $d^{\prime}$ " aide au retour " 
consistent premièrement en une " assistance financière " d'un montant de $1000 \mathrm{CHF}(900 €)$ par adulte et $500 \mathrm{CHF}(450 €)$ par enfant. Ces prestations comportent également une " assistance matérielle " pour la réalisation d'un projet de réintégration financé sur place, par I'intermédiaire de I'OIM, jusqu'à un montant de $3000 \mathrm{CHF}(2700 €)$ par famille ${ }^{15}$. II est à noter que le montant de ces prestations peut être revu à la hausse dans le cas de la mise en œuvre d'un programme visant les ressortissants d'un pays spécifique ${ }^{16}$. Ces prestations peuvent aujourd'hui bénéficier à toute personne ayant effectué une demande d'asile en Suisse, $n^{\prime}$ ayant pas été l'objet d'inculpation pour des délits graves ou répétés. Elles étaient à leur origine uniquement destinées aux personnes dont la demande d'asile était en cours (ODM et OIM Berne, 2007).

Les statistiques existantes démontrent, par ailleurs, que c'est en tout premier lieu aux personnes dont la demande d'asile a été rejetée que s'adresse en réalité l'aide au retour. Ainsi, selon le dernier rapport d'évaluation de l'aide au retour, $70 \%$ des participants aux programmes d'aide au retour avaient reçu une décision d'asile négative, $29 \%$ avaient une demande d'asile pendante ${ }^{17}$ et seul $1 \%$ des participants avaient reçu une demande d'asile positive (Kessler et al., 2013 : 12). Une autre évaluation réalisée par I'ODM datant de 2005 concernant I'aide au retour démontrait, quant à elle, que le pourcentage de personnes ayant bénéficié d'une aide au retour était constitué à plus de $80 \%$ par des requérants d'asile déboutés en 2003 et 2004 (ODM, 2005 : 12). Ainsi, même si en théorie, I'aide au retour peut également bénéficier à des personnes ayant obtenu l'asile en Suisse, la très grande majorité des retours ayant lieu dans ce cadre est le fait de requérants d'asile déboutés.

Le " slogan " de l'aide au retour figurant sur l'entête des documents de présentation des programmes - "Asile : si c'est non que faire ? " - signifie par ailleurs clairement la nouvelle orientation de l'aide au retour à l'égard des requérants déboutés. La réorientation de l'aide au retour à l'égard non plus des personnes en cours de procédure, mais de ceux dont la demande d'asile avait été rejetée s'était d'ailleurs traduite au niveau institutionnel en octobre 2008, par le transfert de la "Section aide au retour " de la "Division Séjour " de I'ODM, à la "Division Retour ", également responsable pour les " retours sous contrainte".

L'orientation très claire de l'aide au retour à l'égard de personnes n'ayant pas le droit de demeurer sur le territoire suisse nous rappelle que les départs ayant lieu dans ce cadre ne devraient - dans leur très grande majorité - pas être qualifiés de "volontaire ". L'emploi abusif de ce qualificatif permet de maintenir I'idée selon laquelle l'assistance matérielle représente - pour les personnes concernées - une véritable alternative aux " départs sous contrainte ". Cela facilite à son tour la communication au sujet des pratiques gouvernementales controversées, impliquant un recours à la coercition.

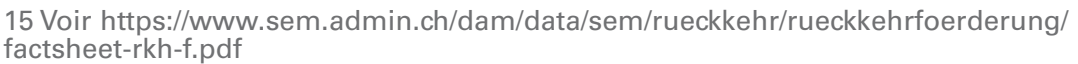

16 Dans le cas du programme destiné aux ressortissants nigérians, le montant de l'assistance matérielle avait été doublé. Actuellement, des programmes spécifiques d'aide au retour sont en cours pour les ressortissants de Guinée, du Nigeria et de Tunisie.

17 Parmi ce pourcentage relativement élevé figurent toutefois également les personnes ayant fait un recours après un premier rejet de leur demande d'asile. 
Un autre enjeu permettant d'expliquer la prégnance de la catégorie de " retour volontaire " dans le cadre de " I'aide au retour " est l'implication de l'OIM, dont les bureaux nationaux coordonnent le retour des requérants déboutés. Elle est également responsable de la mise en œuvre et du suivi des microprojets individuels de réinsertion. La charte constitutive de I'OIM limite en effet les activités de cette organisation à fournir des services aux États dans le cadre de " retour volontaire ". Elle a de ce fait adopté une définition extrêmement large des "retours volontaires" en qualifiant de "retours volontaires sous contraintes " le retour de personnes " n'ayant plus de droit au séjour dans le pays d'accueil et choisissant de rentrer dans leur pays d'accueil de leur propre volonté " (OIM, $2004: 10)^{18}$. L'inclusion du retour des personnes ne bénéficiant pas d'autorisation de séjour à l'intérieur de la catégorie de " retour volontaire " permet de ce fait à I'OIM d'être la principale organisation chargée de la mise en œuvre - et bien souvent de l'évaluation - des " programmes de retours volontaires assistés ".

\section{Contrainte juridique}

II semble premièrement nécessaire de rappeler que, malgré le discours mettant en avant le caractère " volontaire " ou encore " autonome " d'une majorité de départs, c'est le rejet de la demande d'asile et la décision de renvoi concomitante qui fondent le point de départ de l'ensemble de la politique de retour suisse. Ainsi, le fait que les départs aient lieu sans qu'il ne soit fait un recours effectif à la contrainte physique ou encore dans le cadre d'un programme d'assistance au retour ne peut en aucun cas faire oublier l'obligation légale de quitter le territoire qui pèse sur les individus et les différentes formes de contraintes qui en découlent.

Dès lors que les ressortissants étrangers ne disposent pas d'une autorisation légale de séjour sur le territoire, la contrainte légale qui les enjoint à quitter le territoire devrait donc exclure tout emploi du qualificatif de "volontaire " pour décrire ces retours. Même si les modalités par lesquelles la décision de renvoi est exécutée peuvent impliquer un certain degré de consentement de I'individu, celui-ci ne peut en aucun cas être considéré comme une décision librement consentie de retourner dans leur pays d'origine. En effet, le fait qu'une contrainte légale préside à l'ensemble des procédures mises en œuvre par les autorités devrait, selon la définition même qu'en donnent les responsables de cette politique, exclure l'usage d'un terme qui véhicule l'idée d'une décision librement consentie.

Il est de ce fait important de rappeler que ce n'est - pour l'immense majorité des cas - qu'une fois que les personnes ont épuisé toutes les voies légales leur permettant de demeurer en Suisse, qu'elles sont prêtes à considérer l'option d'un retour assisté. On constate donc aisément l'importance de la contrainte juridique pour l'ensemble des personnes concernées par la politique de retour, y compris celles qui finissent par accepter de rentrer dans le cadre d'un programme de retour assisté. II apparaît donc nécessaire, d'emblée, de "replacer l'église au milieu du village " en soulignant que l'obligation de quitter le territoire constitue le point de départ, dont découleront les autres formes de contraintes.

18Traduction des auteurs. 


\section{Contraintes matérielles}

L'importance que revêt la contrainte juridique liée à l'obligation de quitter le territoire est par ailleurs intimement liée aux contraintes matérielles qui pèsent sur les requérants déboutés. En ne considérant que des facteurs purement économiques, l'assistance proposée dans le cadre de l'aide au retour ne peut avoir un caractère incitatif que dans la mesure où les personnes estiment avoir de meilleures perspectives en rentrant dans leur pays d'origine à la faveur d'un programme de retour assisté qu'en demeurant sur le territoire. Partant de cette perspective particulièrement étroite, diverses mesures ont été prises afin de péjorer les conditions de vie des requérants déboutés.

La première porte sur l'interdiction de travailler faite aux requérants d'asile déboutés. Une assistance d'un montant de $4000 \mathrm{CHF}$ (3 $600 €$ ) - ou même dans le meilleur des cas de $7000 \mathrm{CHF}(5600 €)$, comme c'est le cas pour les programmes spécifiques à destination du Nigéria - n'a vraisemblablement qu'un effet incitatif très limité pour des personnes disposant d'une autorisation de séjourner et de travailler en Suisse, et pour qui cette somme ne représente a priori guère plus de deux salaires mensuels ${ }^{19}$. Même pour des individus pouvant espérer trouver un travail au noir, l'aide au retour ne peut constituer qu'une incitation relativement modeste, représentant l'équivalent de deux à quatre mois de salaires ${ }^{20}$. On peut donc ici clairement constater qu'elle ne peut vraisemblablement avoir un effet incitatif que pour des personnes n'ayant pas ou peu de perspectives de trouver un emploi en Suisse et se trouvant par conséquent dépendantes de l'assistance qui leur est fournie dans le cadre de l'asile ${ }^{21}$.

Selon la logique dissuasive visant à péjorer les conditions de vie des requérants déboutés afin de les pousser à quitter le territoire, une série de mesures ont été prises au cours des années 2000. En effet, jusqu'en 2004, tous les requérants d'asile dont la demande avait été rejetée bénéficiaient de la même assistance sociale que celle à laquelle ils avaient droit durant leur procédure $\mathrm{d}^{\prime}$ asile, et ce jusqu'à leur éloignement effectif ${ }^{22}$. Certains des bénéfices attachés à la procédure d'asile perduraient ainsi au-delà de la décision négative pour ne se terminer en réalité qu'avec l'expulsion du territoire. II apparaissait alors logique, d'un point de vue pragmatique, de continuer à fournir une assistance à des personnes dépourvues de ressources et d'autorisation de travailler, et cela tant que durerait leur présence en Suisse.

19 Selon l'Office fédéral de la statistique suisse, en 2012, le salaire brut au-dessous duquel se trouvent $25 \%$ des personnes les moins bien payées (interquartile 1) se situe en effet à $4450 \mathrm{CHF}(4011 €)$.

20 Dans leur étude Flückiger et Pasche (2004 : 15) font en effet état d'un salaire brut moyen pour les travailleurs sans-papiers de l'économie domestique équivalant à 2176 CHF par mois (3 $734 €$ ).

21 Bien que les coûts liés à l'exécution d'une procédure de renvoi ou du maintien des personnes au sein des structures d'assistance soient beaucoup plus onéreux, les autorités sont réticentes à augmenter les montants octroyés par peur de créer " l'appel d'air " auprès des requérants d'asile potentiels. Le paradoxe de la politique d'aide au retour consiste donc dans le fait qu'elle devrait constituer une incitation au départ, mais ne devrait pas pour autant inciter à venir demander l'asile en Suisse dans le seul but de pouvoir en bénéficier.

22 L'assistance sociale attribuée aux personnes du domaine de l'asile est d'un tiers inférieure aux prestations d'aide sociale dont peuvent bénéficier les Suisses et les étrangers ayant une autorisation de séjour en Suisse (Efionayi-Mäder et al., 2012). 
Cette logique pragmatique d'assistance fera place à une logique dissuasive lorsqu'en 2004, il sera décidé de supprimer l'aide sociale aux requérants déboutés. Le raisonnement sous-jacent à cette décision politique consistait à penser que les requérants déboutés seraient incités à retourner dans leur pays d'origine si les conditions matérielles qui leur étaient offertes en Suisse ne correspondaient pas à leurs attentes. Cet " effet dissuasif " sur les requérants déboutés est le plus souvent véhiculé au travers du discours sur la " réduction de l'attractivité " de la Suisse pour les personnes n'ayant pas un réel besoin de protection (DFJP, 2012 : 4). La suppression de l'assistance sociale a donc été explicitement présentée comme ayant un caractère dissuasif dont le but avoué était d'inciter les personnes n'ayant pas de réels besoins de protection à quitter d'elles-mêmes la Suisse.

La logique dissuasive de réduction de l'attractivité du système d'asile suisse a été quelque peu contrecarrée par la Constitution Fédérale Suisse, dont l'article 12 garantit à toute personne présente sur le territoire un minimum vital pour pouvoir vivre dans la dignité ${ }^{3}$. Suite à la suppression de l'assistance sociale, des dispositifs d'" aide d'urgence " au sein des cantons se sont alors mis en place (Sanchez-Mazas, 2011). Toutefois la même logique de dissuasion a prévalu à l'instauration de cette aide comme le démontrent les propos d'un responsable cantonal:

"L'aide d'urgence, c'est quand même une mesure qui a pour but de les précariser pour les amener à collaborer à leur départ " (Responsable d'un service cantonal de migration, entretien du 12 juin 2013).

Les cantons ont ainsi institué des systèmes qui - tout en fournissant une aide minimale garantie par la Constitution - permettaient malgré tout de dissuader les requérants déboutés à demeurer sur le territoire. Le canton de Genève a choisi, par exemple, de ne pas délivrer une assistance en espèces, mais en nature sous forme de "plateau repas " ôtant par conséquent aux individus jusqu'à leur capacité de décision quant à la nourriture qu'ils pourront ingérer durant leur séjour en Suisse. Lorsqu'une aide en espèces est versée, elle s'élève en moyenne à $9 \mathrm{CHF}(8 €)$ par jour ${ }^{24}$.

La logique dissuasive de précarisation des conditions de séjour se retrouve également au niveau des conditions d'hébergement offertes aux requérants déboutés. Le canton de Lausanne optait, par exemple, pour loger les individus dans des abris souterrains de la protection civile au sein desquels les personnes ne pouvaient demeurer que de $17 \mathrm{~h}$ à $8 \mathrm{~h}$ du matin. Le canton de Zürich optait, quant à lui, pour un " système de rotation " visant à empêcher les individus de nouer des liens au sein des centres ou en dehors en exigeant de la part de requérants déboutés de changer de centre d'hébergement chaque semaine. D'autres cantons, tels que le canton de Berne ou des Grisons, choisiront des zones montagneuses et difficilement accessibles pour y placer les centres d'aide d'urgence.

23 "Quiconque est dans une situation de détresse et n'est pas en mesure de subvenir à son entretien a le droit d'être aidé et assisté et de recevoir les moyens indispensables pour mener une existence conforme à la dignité humaine".

24 À titre indicatif, un café vaut en Suisse environ $3 \mathrm{CHF}(2,7 €)$. 
La détérioration des conditions de vie des requérants déboutés vise donc à dissuader les personnes qui n'auraient pas de réels besoins de protection à préférer quitter le territoire que d'y rester vivre les conditions qui leur sont offertes. Cette "réduction de l'attractivité » de la Suisse et de son système d'asile permettrait également aux prestations offertes dans le cadre de l'aide au retour de constituer une incitation au retour. La logique dans laquelle est pensée l'incitation matérielle au départ se rapporte donc bel et bien à ce double mouvement consistant, d'une part, à détériorer les conditions d'existence des requérants déboutés, pour, de l'autre, leur offrir une assistance matérielle, qui sans cela ne pourrait pas constituer une quelconque incitation.

\section{Contraintes psychologiques ou le recours indirect de la contrainte physique}

En parallèle à cette combinaison de contraintes et d'incitations d'ordre matériel, les autorités usent également de moyens de contraintes psychologiques consistant à menacer la personne d'un recours à la contrainte physique si elle ne partait pas d'elle-même. Ainsi, après le rejet de leur demande d'asile, les autorités cantonales informent les déboutés qu'ils font l'objet d'une décision de renvoi et qu'ils sont par conséquent dans l'obligation légale de quitter le territoire suisse. Afin d'inciter les requérants déboutés à ne pas opposer de résistance lors de l'exécution de leur décision de renvoi, les autorités leur expliquent que s'ils collaborent avec les autorités, ils pourront quitter le territoire au bénéfice d'une aide au retour. Toutefois, en cas d'absence de collaboration, il sera alors fait recours aux forces de police afin de placer la personne en détention administrative, puis de la mettre dans un avion spécialement affrété (" vols spéciaux ") où les individus peuvent être entièrement entravés. L'usage de cette " stratégie de la carotte et du bâton " est explicitement admis par les responsables de l'exécution du renvoi, qui admettent également que l'aide au retour n'aura pas d'effet, sans l'éventualité du recours à la coercition.

"C'est vrai que si on a la " carotte " du départ volontaire, ça marche pas mal si on a le "bâton" du renvoi sous contrainte, ça marche mieux. On peut dire à la personne : "Écoutez soit vous rentrez librement avec une aide au retour, ou bien le renvoi forcé sans rien du tout !" "(Responsable d'un service cantonal de migration, entretien du 05 août 2013).

Les requérants déboutés sont par conséquent informés le plus rapidement et clairement possible que, dans le cas où ils refuseraient de rentrer " volontairement " dans le cadre des programmes d'aide au retour, ils seront immanquablement renvoyés par la force. Concomitamment aux informations dispensées sur les formes d'assistance disponibles dans le cadre de l'aide au retour, les autorités cantonales insistent donc également sur les mesures qui seront prises à leur égard dans le cas où elles n'accepteraient pas de rentrer. L'administration fédérale informe, notamment à l'aide du dépliant reproduit ci-dessous, les requérants fraichement déboutés sur les procédures progressivement mises en œuvre au cas où ils refuseraient de quitter le pays de manière " volontaire ". 


\section{Figure 1 : Dépliant remis aux requérants d'asile lors de la réception d'une décision négative}

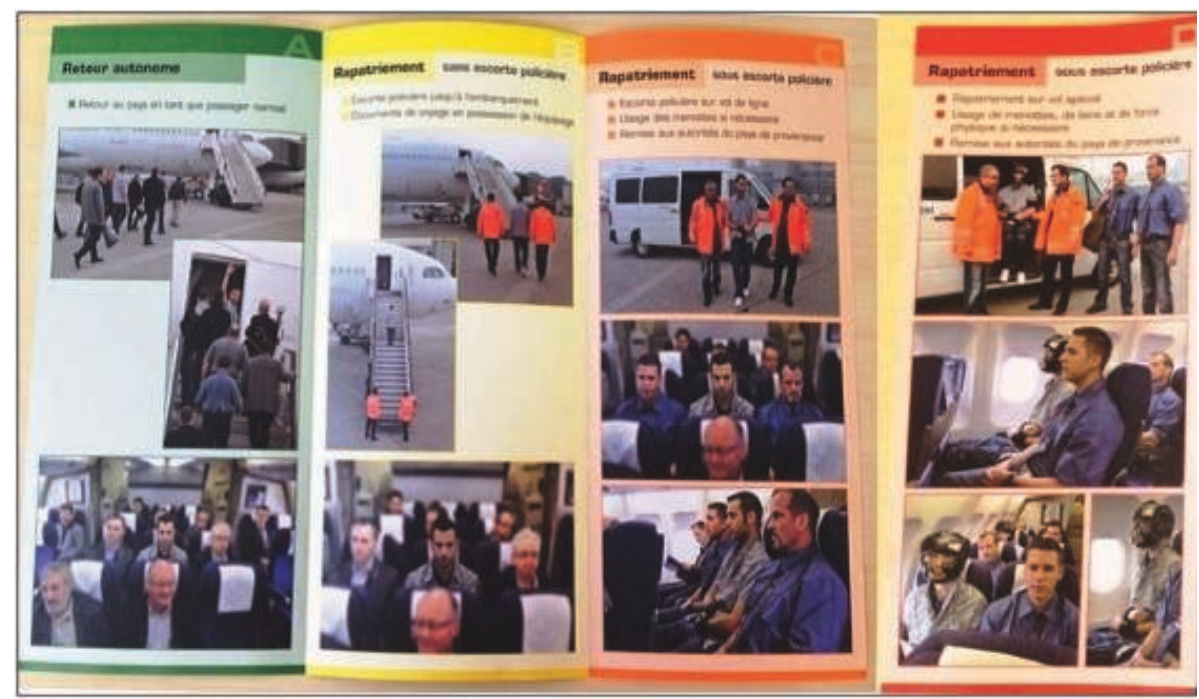

Légende du dépliant:

A. Retour autonome : Retour au pays en tant que passager normal.

B. Rapatriement sans escorte policière : Escorte policière jusqu'à l'embarquement.

Documents de voyage en possession de l'équipage.

C. Rapatriement sous escorte policière : Escorte policière sur vol de ligne. Usage des menottes si nécessaire. Remise aux autorités du pays de provenance.

D. Rapatriement sous escorte policière : Rapatriement sur vol spécial. Usage de menottes, de liens et de force physique si nécessaire. Remise aux autorités du pays de provenance.

Ce dépliant illustre la manière dont la gradation de la contrainte est présentée aux étrangers faisant l'objet d'une décision de renvoi. II démontre ainsi particulièrement bien l'effet d'incitation au départ " autonome " que cherchent à produire les autorités en illustrant ce qui attend les personnes qui refuseraient de quitter le territoire. II ne constitue que la première annonce des conséquences d'un refus de collaboration dans le cadre de leur procédure de renvoi. Une pression de plus en plus forte sera mise sur les requérants déboutés afin qu'ils effectuent des démarches en vue de leur départ. Ainsi, si après quelques semaines la personne n'a toujours pas entrepris les démarches en vue d'un retour "volontaire ", les autorités cantonales commenceront alors à menacer l'individu de recourir à la détention administrative et aux forces de police afin de mettre le renvoi à exécution. De nombreux témoignages de requérants déboutés font état de la pression croissante que les autorités exercent sur les individus, et de ce que cela provoque chez ces personnes: 
"La dernière fois, ils m'ont retenu avant de me donner le tampon25, et m'ont fait monter au bureau. Là-bas, ils m'ont dit que je n'avais plus rien à faire ici, que j'étais illégal, que je devais quitter la Suisse... que si je n'organisais pas mon départ en allant à la Croix-Rouge genevoise et à mon ambassade, ce serait la police qui allait me renvoyer! Ce jour-là, j'ai cru que ma tête allait exploser. Et la prochaine fois, je suis sûr qu'ils vont me retenir encore... " (Requérant d'asile débouté attribué au canton de Genève, entretien réalisé en août 2007).

L'usage indirect des formes de contraintes en tant que " moyen de pression " sur les requérants déboutés est loin d'être une pratique officieuse à laquelle recouraient certains fonctionnaires peu scrupuleux au sein des services cantonaux. La citation suivante démontre au contraire que cet usage indirect de la contrainte est explicitement admis comme l'une des principales fonctions des mesures de contraintes. "Les représentants des autorités ainsi que les autres personnes interrogées ont souligné que les systèmes d'incitation peuvent contribuer à motiver les personnes en situation irrégulière à retourner dans leur pays. Dans ce contexte, les mesures de contrainte servent essentiellement de moyen de pression pour les personnes qui préfèrent séjourner illégalement en Suisse plutôt que de faire appel à l'aide au retour " (Contrôle parlementaire de l'administration, $2005: 40$ ).

Ainsi, nous pouvons constater que malgré un usage direct limité de la contrainte physique par les autorités suisses, la place que celle-ci occupe au sein de la politique de retour est en réalité primordiale. En effet, plus encore que son usage effectif, c'est la potentialité d'y faire recours qui constitue la pierre angulaire de l'ensemble de la politique de retour. Le fait que, d'après les déclarations, même des plus hauts responsables de l'administration, l'ensemble de la politique de retour repose sur la possibilité d'user la contrainte physique amène encore une fois à remettre en question le caractère volontaire des retours après une décision de renvoi. Même lorsque le départ intervient sans qu'il soit fait un usage direct de la contrainte physique, c'est la menace d'y recourir qui permet, en partie au moins, d'expliquer que les requérants déboutés acceptent de rentrer de manière " volontaire " ou " autonome ". C'est ce qui ressort de nombreux entretiens effectués avec des requérants déboutés rentrés dans le cadre d'un programme d'aide au retour.

"I had no intention to come back, but the authorities told me to come back. [...] I even said that I won't sign for this return assistance, but they told me: look, you better sign and take return assistance, or we will arrest you, chain your hands to your back and move you to the country where you come from " (Requérant d'asile débouté rentré au Nigéria dans le cadre d'un programme d'aide au retour, entretien réalisé en janvier 2010).

La potentialité de l'usage de la contrainte physique, dans le cas des expulsions " niveau 4 ", constitue donc avant tout une technique de gouvernement permettant aux autorités de faire pression sur les requérants d'asile déboutés afin de les contraindre à quitter la Suisse de manière " autonome ". On constate donc la manière dont diverses formes de contraintes légales, matérielles et psychologiques sont combinées avec l'incitation matérielle que représente l'aide

25 II s'agit du tampon effectué par les autorités cantonales, qui permet aux individus d'avoir accès à l'aide d'urgence. 
au retour afin d'inciter les individus à collaborer à leur renvoi, ou du moins à ne pas opposer de résistance physique.

Un entretien effectué par Estelli (2015 : 91) avec Karl Lorenz ${ }^{26}$, un ancien responsable lors de la mise en place de l'aide au retour dans les années 1990 par la Confédération, montre clairement que la stratégie consistant à favoriser les retours "volontaires " en posant le retour sous contrainte comme unique autre alternative a été pensée comme telle dès l'origine de la politique :

"On était de l'avis, comme aujourd'hui, que le retour volontaire marche uniquement s'il y a aussi la possibilité de retour sous contrainte. Si les gens peuvent choisir entre rentrer volontairement et rester en Suisse, je pense que le choix est plutôt clair, on va plutôt opter pour le séjour en Suisse, pour rester en Suisse. Si le choix est plutôt de rentrer volontairement ou d'être renvoyé sous contrainte, je pense que là on a la chance que les gens acceptent un retour volontaire " (Entretien du 23 octobre 2014)27.

Ainsi, le choix qui est offert aux individus ne porte évidemment pas sur le fait de savoir s'ils veulent demeurer sur le territoire ou non, mais uniquement sur la manière par laquelle leur renvoi sera effectivement mis à exécution. Cela équivaut en réalité à ne laisser aux requérants déboutés qu'une décision qui devrait a priori s'imposer d'elle-même, à savoir s'ils préfèrent quitter la Suisse avec quelques milliers de francs ou des menottes.

C'est donc dans l'articulation de différentes formes de contraintes (juridiques, matérielles, psychologiques et physiques) combinée à l'incitation matérielle que représente l'aide au retour que réside la stratégie mise en œuvre par les autorités suisses afin de dissuader les personnes à demeurer sur leur territoire. II apparaît dès lors clairement que le fait que l'exécution des décisions de renvoi intervienne sans qu'il ne soit finalement fait recours effectif à la coercition physique ne peut pas pour autant amener ces retours à être considérés comme " volontaires " ou même " autonomes ". Plutôt qu'une alternative au retour sous contraintes, les instruments des programmes d' " aide au retour " devraient être clairement identifiés comme faisant partie intégrante de l'ensemble d'une politique visant à gouverner le comportement d' "étrangers indésirables ", afin de les pousser à se plier à l'obligation légale de quitter le territoire. La rationalité de gouvernement que nous qualifions $d^{\prime}$ " expulsions volontaires " consiste donc à mettre en place des conditions telles que les étrangers se soumettent d'eux-mêmes à l'injonction de quitter le territoire qui leur est faite, sans que les autorités aient à faire un usage (coûteux et contesté) de la coercition physique.

26 Ancien chef de la section d'aide au retour à I'ODM et ex-directeur du bureau national de l'OIM à Berne.

27 À noter que cet extrait illustre parfaitement une confusion qui existe dans I'usage du terme " retour volontaire " qu'on retrouve souvent. Dans un premier temps, l'interviewé le met en relation avec la possibilité de pouvoir rester en Suisse et par la suite avec le renvoi forcé. Ce faisant, il omet clairement que le fait d'opter pour le retour dit " volontaire " sous la menace ou la pression d'un renvoi forcé ne relève pas d'une décision librement consentie. 


\section{Conclusion}

L'objectif de cette contribution réside dans la mise en évidence d'une rationalité de gouvernement des "étrangers indésirables " désignée sous le terme $\mathrm{d}^{\prime}$ " expulsions volontaires ". Cette logique de gouvernement vise à exécuter les renvois d'étrangers en les amenant à se plier à la décision des autorités de renvoi, afin d'éviter (autant que possible) de faire un recours direct à la coercition physique.

Pour mettre en évidence cette gouvernementalité, nous avons commencé par remettre en question le discours officiel entourant et légitimant ce qui est connu en Suisse sous le terme de "politique de retour ". Comme cela a été démontré, l'objectif avoué de cette politique consiste à mettre à exécution les décisions de renvoi des personnes se trouvant illégalement en Suisse, avec un focus particulier sur les requérants d'asile déboutés. Si le qualificatif de " politique d'exécution des renvois " apparait déjà nettement plus fidèle au but poursuivi, le concept $d^{\prime}$ " expulsion volontaire " nous semble utile afin de souligner plus spécifiquement la manière dont la mise en œuvre des décisions d'expulsion est pensée.

Nous avons mis l'accent sur la catégorisation binaire entre " retours volontaires " et " contraints " sur laquelle repose en bonne partie le discours officiel légitimant cette politique. En nous appuyant sur les autres formes de catégorisation légales et administratives, ainsi que sur des observations et entretiens avec les acteurs concernés, nous avons pu démontrer comment cette catégorisation binaire amène à masquer les diverses formes de contraintes légales, matérielles, et psychologiques qui sous-tendent toutes procédures d'exécution de renvoi, et cela même lorsque le départ intervient sans qu'il soit fait usage de la force.

Nous souhaitons que cette analyse contribue à renoncer à la catégorie de " retour volontaire ", afin de qualifier tout départ ayant lieu après qu'une décision de renvoi ait été prononcée, et ce même s'il intervient dans le cadre d'un programme d'assistance. Le maintien de cette terminologie semble, au moins partiellement, consciemment entretenu par des instances gouvernementales et intergouvernementales souhaitant véhiculer une image plus favorable d'une politique suscitant de nombreux débats, notamment au sein de la société civile. Il est indéniable qu'un départ ayant lieu dans le cadre d'un " programme d'aide au retour " est préférable pour l'ensemble des acteurs concernés. Le choix offert aux personnes concernées par une décision d'expulsion ne porte évidemment pas sur le fait de rester ou non sur le territoire, mais uniquement sur les conditions du déroulement de l'exécution du renvoi. À ce titre les départs ayant lieu dans ce cadre ne peuvent pas être considérés comme une " alternative " aux renvois, mais bien comme la moins " mauvaise " des formes de "départs sous contraintes ". Le fait que ces départs ne puissent pas être considérés comme une alternative au renvoi devrait également amener à renoncer à légitimer le recours à la coercition physique à l'encontre des personnes ayant refusé le " choix " de quitter " volontairement " qui leur avait été fait.

Un dernier élément du discours sur la politique de retour, afin de mettre en évidence un mode de gouvernement particulier des " étrangers indésirables ", porte sur la manière dont ce discours légitime l'usage de la contrainte 
physique en insistant, d'une part, sur la nécessité absolue de son maintien pour l'ensemble de la politique, et de l'autre, sur l'usage très restreint qui en est fait. En effet, même dans le cas où il n'est pas fait un recours direct à des formes de contraintes physiques, nous avons fait ressortir la manière dont la potentialité d'y faire recours constitue le socle sur lequel repose toute cette politique d'exécution des renvois.

La considération de l'ensemble de ces éléments permet au final de révéler une rationalité de gouvernement visant à produire le comportement désiré de la part d'une catégorie de la population, à savoir la collaboration des requérants déboutés à leur procédure de renvoi. Ainsi, l'illégalisation du séjour, l'interdiction de travail, la suppression de l'aide sociale et les menaces du recours à la détention administrative et aux "vols spéciaux " constituent autant de contraintes, qui - en conjonction avec l'incitation matérielle que représente I'aide au retour - visent à procéder à l'exécution des renvois, en évitant autant que possible faire un recours effectif aux formes de contraintes physiques. Ce mode de gouvernement consiste donc en une articulation de différentes formes de contraintes et d'incitations visant à pousser les " étrangers indésirables " à quitter le pays. Ces dernières sont associées les unes aux autres avec l'objectif de produire le comportement désiré, à savoir le départ de l'étranger "indésirable ".

Avant de conclure, il est nécessaire de préciser que si cette contribution a cherché à mettre en lumière une rationalité de gouvernement des " étrangers indésirables ", elle ne s'est toutefois pas attachée à évaluer dans quelle mesure cette gouvernementalité est effectivement efficace afin de produire le comportement attendu. Bien qu'il n'existe pas en Suisse de statistiques sur le taux de non-exécution des décisions de renvoi, de nombreux éléments semblent toutefois suggérer que la politique mise en œuvre par la Confédération se heurte à de nombreux obstacles. Une étude portant sur les requérants d'asile déboutés en Suisse a notamment souligné les mécanismes psychosociaux de résistance (en particulier ceux de réactance et de résilience) qu'induisait chez les requérants l'étau de contraintes dans lesquelles l'État les place (Sanchez-Mazas, 2011). En outre, alors que la grande majorité des instruments de la " politique de retour " décrite ici porte sur l'objectif de contraindre l'étranger aux décisions de l'État suisse, l'aboutissement de l'exécution du renvoi dépend d'une multiplicité d'acteurs et de facteurs, sur lesquels les autorités suisses n'ont parfois que peu d'influence ${ }^{28}$. Si l'importance de la collaboration des États d'origine des étrangers dans le cadre de cette procédure a été relevée (Ellerman, 2008) et que les États européens cherchent également à prendre des mesures pour s'assurer leur collaboration, les résultats demeurent en dessous de leur attente. Les difficultés d'exécution des décisions de renvoi ne sont d'ailleurs pas spécifiques à la Suisse. La non-application des décisions d'expulsion est un phénomène répandu, qui a donné lieu à diverses analyses, telles que celle du deportation gap (Gibney, 2008), interprétant l'écart entre le nombre de décisions d'expulsions notifiées et

28 " Les résultats de l'enquête ont également montré qu'un renvoi ne dépend pas seulement de l'application ou du durcissement des mesures de contrainte, mais également de facteurs externes, non influençables, tels que le refus de certains États de reprendre leurs ressortissants rapatriés sous contrainte ", Contrôle parlementaire de I'administration (2005: 2, en italique dans I'original). 
celui d'expulsions effectives. II s'agit également d'un problème qui préoccupe de plus en plus la Commission européenne (2014) comme le montre son livre vert sur la politique de I'UE en matière de retour. Selon son commissaire chargé de la migration, Dimitris Avramopoulos (2014), le taux d'application des décisions d'expulsion prises par les pays de l'UE n'était que $39 \%$ en 2014.

II pourrait être particulièrement intéressant à l'avenir d'analyser quelles sont les résistances qu'opposent les personnes menacées d'expulsion ou encore les États d'origine des migrants à la mise en œuvre effective de cette gouvernementalité. Par ailleurs, il serait également opportun de chercher à voir s'il est possible de retracer l'origine de cette forme de gouvernement des " étrangers indésirables " au sein d'autres pays d'Europe ou d'Amérique du Nord, ainsi que d'attester d'une éventuelle diffusion de cette logique au niveau de l'UE.

\section{Références bibliographiques}

Avramopoulos Dimitris (2014) Letter to Home Affairs and Ministers of the EU countries, Brussels, 01 06. 2015, Ares(2015) 2397724, 11 p., [online] last checked on 15/09/2015. URL: http://www.statewatch.org/news/2015/jun/eu-AVRAMOPOULOS-migration-letter-to-eu-ministers.pdf

Blitz Brad K., Sales Rosemary and Marzano Lisa (2005) Non-Voluntary Return? The Politics of Return to Afghanistan, Political Studies, 53, pp. 182-200.

Bröckling Ulrich, Krasmann Susanne and Lemke Thomas (2011) From Foucault's lectures at the Collège de France to the studies of governmentality, in Governmentality, in Ulrich Bröckling, Susanne Krasmann and Thomas Lemke Eds., Current Issues and Future Challenges, New York and London, Routledge, pp. 1-33.

Commission européenne (2014) Communication de la Commission au Conseil et au Parlement européen sur la politique de l'Union européenne en matière de retour, $\operatorname{COM(2014)} 199$ final, Bruxelles.

Contrôle parlementaire de l'administration (2005) Évaluation des mesures de contrainte en matière de droit des étrangers, Rapport final du Contrôle parlementaire de l'administration à l'attention de la Commission de gestion du Conseil national du 15 mars 2005, Berne, 76 p.

DFJP (2012) Guide DFJP Rapatriements dans les domaines des étrangers et de I'asile, Office fédéral des migrations Division Procédure à la centrale et retour, Berne-Wabern, $34 \mathrm{p}$.

Efionayi-Mäder Denise, Stünzi Robin et de Senarclens Clément (2012) Soumettre tous les requérants d'asile au régime de l'aide d'urgence? Données utiles au débat public, Neuchâtel, Centre de droit des migrations, [en ligne] consulté le 13/04/2015. URL : http://www.unine.ch/files/live/sites/ius-migration/files/ Questions_Réponses.pdf

Ellermann Antje (2008) The limits of unilateral migration control: Deportation and inter-state cooperation, Government and Opposition, 32 (2), pp. 168-189.

Estelli Mélanie (2015) La politique d'aide au retour. L'exemple de la Suisse, Mémoire de licence, Institut de géographie de I'Université de Neuchâtel, Neuchâtel, 128 p. 
Fassin Didier (2011) Policing Borders, Producing Boundaries. The Governmentality of Immigration in DarkTimes, Annual Review of Anthropology, 40, pp. 213-226.

Flückiger Yves et Pasche Cyril (2004) Analyse du secteur clandestin de l'économie domestique à Genève, Observatoire Universitaire de I'Emploi, Genève, 39 p., consulté le 13/04/2015.

Foucault Michel (2004) Sécurité, territoire, population, Cours au Collège de France, 1977-1978, Paris, Gallimard/Seuil, 435 p.

Foucault Michel (1994a) La "gouvernementalité ", in Michel Foucault, Dits et écrits, Paris, Gallimard, vol. III, pp. 635-657.

Foucault Michel (1994b) Les techniques de soi, in Michel Foucault, Dits et écrits, vol. IV, Paris, Gallimard, pp. 783-813.

Geiger Martin and Pécoud Antoine (Eds.) (2013) Disciplining the Transnational Mobility of People, New-York, Palgrave Macmillan, 271 p.

Gibney Matthew J. (2008) Asylum and the Expansion of Deportation in the United Kingdom. Government and Opposition, 43 (2), pp. 146-167.

Gibney Matthew J. and Hansen Randall (2003) Deportation and the liberal state: the forcible return of asylum-seekers and unlawful migrants in Canada, Germany and the United Kingdom, New Issues in Refugee Research, Working Paper 77, Genève, UNHCR, EPAU, 21 p.

Inda Jonathan and Dowling Julie (2013) Governing Immigration Through Crime, Stanford, Stanford University Press, 320 p.

Kaser Eric et Schenker Saskia (2008) L'aide au retour de la Suisse : bilan et perspectives, Annuaire suisse de politique de développement, 27 (2), pp. 199-212.

Kessler Daniel, Zürcher Dieter and Wigger Fiona (2013) Assisted Voluntary Return and Reintegration: An External Evaluation. KEK-CDC and BSS Consultants, in Bericht des Bundesrats in Erfüllung des Postulats Müller Philipp 11.3062 vom 8. März 2011 Wirksamkeit und Kosten der Rückkehrhilfe, last checked on 13/04/2015.

Koser Khalid (2001) The return and reintegration of rejected asylum seekers and irregular migrants: an analysis of government assisted programs in selected European countries, Genève, IOM, $47 \mathrm{p}$.

Lascoumes Pierre (2004) La Gouvernementalité : de la critique de l'État aux technologies du pouvoir, Le Portique, [en ligne] consulté le 09/05/2012. URL : http:// leportique.revues.org/index625.html

Lemke Thomas (2004) "Marx sans guillemets " : Foucault, la gouvernementalité et la critique du néolibéralisme (traduit de I'anglais par Chemali Marc), Actuel Marx, 36, pp. 13-26.

Noll Gregor (1999) Rejected Asylum seekers:The Problem of Return, International Migration, 37 (1), pp. 267-288.

ODM (2014) Rapport sur la migration 2014, ODM, Bern, 64 p., [en ligne] consulté le 18/10/2017. URL : https://www.sem.admin.ch/dam/data/sem/publiservice/ berichte/migration/migrationsbericht-2014-f.pdf

ODM (2005) Évaluation de l'aide individuelle (directive asile 62.2) : Période du rapport 2001-2004, Berne-Wabern, ODM, 25 p. 
ODM et OIM Berne (2007) 10 ans d'Aide au retour suisse : édition spéciale à I'occasion de I'anniversaire, Going Home : la newsletter de l'aide au retour de I'ODM et de I'OIM Berne, [en ligne] consulté le 21/03/2014. URL : https://www. sem.admin.ch/dam/data/sem/aktuell/newsletters/going_home/2007/goinghome200710-f.pdf

OIM (2007) Glossaire de la migration, Droit international de la migration International, 9, Genève, OIM, 104 p.

OIM (2004) Return Migration: Policies and Practices in Europe, Genève, OIM, $402 \mathrm{p}$.

Sanchez-Mazas Margarita (2011) La construction de l'invisibilité : suppression de l'aide sociale dans le domaine de l'asile, Genève, IES Editions, 304 p.

Walters William (2002) Deportation, expulsion, and the international police of aliens, Citizenship studies, 6 (3), pp. 265-292.

Webber Frances (2011) How voluntary are voluntary returns?, Race\&Class, 52 (4), pp. 98-107. 


\section{Clément de Senarclens et Ibrahim Soysüren}

\section{Les " expulsions volontaires " : un mode de gouvernement des " étrangers indésirables " ?}

Cet article analyse le dispositif d'éloignement des étrangers indésirables en se fondant sur le cas des requérants d'asile déboutés en Suisse. II remet en question le discours selon lequel la " politique de retour " suisse reposerait avant tout sur des mesures d'incitation au départ pour ne faire usage de la contrainte qu'en dernier recours. L'analyse effectuée permet en effet de souligner le rôle de diverses formes de contraintes (juridiques, matérielles et psychologiques), dont usent les autorités dès l'annonce de la décision de renvoi. Ces formes de contraintes président à l'éloignement de ces étrangers même lors de retours qualifiés de "volontaires ". Elle démontre par ailleurs que la contrainte physique apparaît comme l'outil indispensable sur lequel repose l'ensemble du dispositif d'éloignement des étrangers indésirables, et cela même lorsqu'un recours effectif n'est pas fait. Sur la base de cette analyse, l'article vise à mettre en lumière l'existence d'une forme de gouvernement des étrangers indésirables, articulant différentes formes de contraintes et d'incitations en vue d'amener les individus à se soumettre à la décision de renvoi sans que les autorités n'aient à faire un recours effectif à la contrainte physique. Nous désignerons cette gouvernementalité des "étrangers indésirables " par le terme d'" expulsions volontaires ".

\section{"Voluntary Deportations": A Technique of Government of Unwanted Foreigners?}

This article analyses the deportation apparatus of unwanted migrants via the case of rejected asylum seekers in Switzerland. It questions the current political speeches according to which "return policies" are first based on return incentives and would rely on coercition only as a last resort. Indeed, the analysis highlights the importance of the different forms of legal, material and psychological constraints appearing as direct consequences of a deportation order. The crucial role of these constaints should therefore also be recognized when the enforcement of the deportation order occurs in conjunction with the incentive offered within the framework of assisted voluntary return programmes. Indeed, the results of this analysis show that the potential use of physical coercition represents the cornerstone on which all the deportation apparatus is based, even in the case where this most extreme form of constraint does not have to be effectively used. On the basis of these results, the article draw attention to a form of government of unwanted foreigners that aims to submit migrants to deportation orders while at the same time restraining the costly and controversial forms of physical coercition that occurs during the deportation process. This article aims to illustrate this form of government through the notion of "voluntary deportation". 


\section{¿Las «expulsiones voluntarias»: un modo de gubernamentalidad de los extranjeros indeseables?}

Este artículo analiza el dispositivo de deportación de los extranjeros indeseables basándose en el caso de los solicitantes de asilo en Suiza. Cuestiona el discurso que afirma que las "políticas de retorno" se basarían principalmente sobre medidas de incentivos y recurriría a la coacción solo en último recurso. El artículo analiza las diferentes formas de coacción (jurídicas, materiales y psicológicas) utilizadas las autoridades en cuanto se anuncia la decisión de expulsión, aun cuando el regreso es cualificado como "voluntario". El análisis demuestra que la coacción física constituye una de las principales herramientas en la cual se basa el dispositivo de deportación de los extranjeros indeseables, aunque no se ponga siempre en práctica. Basándose en este análisis, el artículo busca demostrar la existencia de una forma particular de gobierno de los extranjeros indeseables. La cual se articula en torno a diferentes formas de coacción y de incentivos que buscan llevar a los individuos a someterse a la decisión de expulsión sin que las autoridades tengan que recurrir a la coacción física de manera efectiva. Se harán referencia a esta gubernamentalidad de los extranjeros indeseables con el término de "expulsiones voluntarias». 